\title{
Préparation des charbons actifs par voie chimique à l'acide phosphorique à base de coque de noix de coco
}

\author{
Clément K. BALOGOUN ${ }^{1 *}$, Moctar L. BAWA ${ }^{2}$, Sèmiyou OSSENI ${ }^{3}$ et Martin AINA ${ }^{4}$ \\ ${ }^{1}$ Laboratoire d'Etude et de Recherche en Chimie Appliquée, Ecole Polytechnique d'Abomey-Calavi, \\ Université d'Abomey-Calavi 01 BP 2009, Cotonou, Bénin. \\ ${ }^{2}$ Laboratoire de Chimie de l'Eau, Faculté des Sciences, Université de Lomé, BP 1515, Lomé, Togo. \\ ${ }^{3}$ Faculté des sciences et Techniques, Université de Parakou (U.P), Bénin. \\ ${ }^{4}$ Laboratoire des Sciences et Techniques de l'Eau, Ecole Polytechnique d'Abomey-Calavi, \\ Université d'Abomey-Calavi 01 BP 2009, Cotonou, Bénin. \\ *Auteur correspondant, E-mail : balclk@yahoo.fr
}

\section{RESUME}

Les matières ligno-cellulosiques sont des précurseurs du charbon actif. Le présent travail a eu pour but la préparation de charbons actifs à partir des coques de noix de coco par activation chimique à l'acide phosphorique. Pour optimiser le procédé de la préparation, les effets des concentrations de l'acide phosphorique $\left(12,5\right.$ à 50\%), des températures d'activation $\left(500\right.$ à $\left.700{ }^{\circ} \mathrm{C}\right)$ et des ratios d'imprégnation Xp de l'acide phosphorique/précurseur $(0,7$ à 2,5$)$, sur la capacité d'adsorption de l'iode ont été étudiés. Les propriétés texturales de ceux-ci ont été déterminées par l'étude d'isotherme d'adsorption d'azote $\mathrm{N}_{2}$. Cette étude révèle que dans les conditions de préparation, la valeur maximale de l'indice d'iode égale à $787 \mathrm{mg} \cdot \mathrm{g}^{-1}$ avec un rendement massique égal à $37,91 \%$ est obtenue pour un ratio d'imprégnation égal à 1,5 , une concentration en acide égale à $50 \%$ et une température de carbonisation égale à $500{ }^{\circ} \mathrm{C}$. Ce charbon actif $($ avec $\mathrm{Xp}=1,5)$ préparé dans les conditions optimisées, a une surface spécifique BET de $1519 \mathrm{~m}^{2} \cdot \mathrm{g}^{-1}$, une distribution des pores à dominance micropores $\left(\mathrm{V}_{\mu} / \mathrm{V}_{\mathrm{T}}=82,4 \%\right)$ et un $\mathrm{pH}_{\mathrm{ZPC}}$ de 3,16 . Les caractéristiques chimiques par analyse spectroscopie FT-IR et la méthode de Boehm ont montré la présence à la surface de ce charbon actif, des groupes carboxyliques, phénoliques et pyrone. Il présente donc une meilleure texture et un caractère acide. (c) 2015 International Formulae Group. All rights reserved.

Mots clés: Charbon actif, coques de noix de coco, activation chimique, ratio d'imprégnation,isotherme d'adsorption, caractéristiques physico-chimiques.

\section{INTRODUCTION}

La gestion des déchets reste un des principaux problèmes dans les pays en développement (PED). La quantité de déchets municipaux est en constante augmentation en raison de l'explosion démographique et de l'urbanisation. Même si les gens sont de plus en plus conscients du problème, celle-ci demeure une préoccupation majeure et permanente des autorités locales. Malheureusement, dans les pays en développement, la voie commune de l'élimination des déchets est la mise en décharge et l'incinération en plein air (COMDEV et AICDD, 2010). Le brûlage en plein air peut affecter la qualité de l'air, 
provoquant ainsi des problèmes de santé publique et contribuer également au réchauffement climatique (Chitou, 2003). Le compostage pourrait constituer une alternative crédible s'il y a une fraction biodégradable appréciable dans les déchets. Mais il existe d'autres possibilités de réutilisation des déchets solides municipaux telles que l'élaboration de charbon actif à partir des résidus ligno-cellulosiques. Ce matériau peut être utilisé dans le traitement des eaux, l'élimination des gaz, comme support dans les phénomènes de catalyse, etc. Parmi les nombreux matériaux considérés comme les plus prometteurs pour l'élimination des micropolluants organiques et minéraux, on cite préférentiellement les charbons actifs (Guo et Rockstraw, 2007).

Différentes études ont été consacrées à la production des charbons actifs à partir de résidus ligno-cellulosiques: les coques de noix de pécan (Guo et Rockstraw, 2007), d'amande (Martínez de Yuso et al., 2014), de noix de coco (Sartape et al., 2012; Cazetta et al., 2013), les noyaux de dattes (Girgis et ElHendawy, 2002), les noyaux d'autres fruits (Aygün et al., 2003; Puziy et al., 2005; Attia et al., 2008), des bois (Budinova et al., 2006; Ahmed et al., 2007), les gousses de Flamboyant Delonixregia (Vargas et al., 2011). C'est dans cette perspective que s'inscrit ce travail dont le but est de valoriser les coques de noix de coco en charbon actif. En effet, au Bénin, les déchets solides des municipalités situées le long de la côte du Golfe de Guinée renferment beaucoup de résidus ligno-cellulosiques. Parmi ceux-ci, on peut citer : la noix de coco, des noix de palme, qui jonchent les rues, à proximité des marchés et dans certaines industries.

Le charbon actif est un matériau carboné solide sous forme granulée ou en poudre noire, ayant une porosité très développée avec la surface interne et la résistance mécanique relativement élevée (Sahu et al., 2010). Il est obtenu soit par activation chimique ou par activation physique. Parmi les activants chimiques, l'acide phosphorique et le chlorure de zinc sont les plus utilisés (Guo et Rockstraw, 2007; Ioannidou et Zabaniotou, 2007; Girgis et ElHendawy, 2002; Tsai et al., 2001). Mais certaines bases telles que $\mathrm{KOH}$ (RomeroAnaya et al., 2014; Basta et al., 2009), $\mathrm{NaOH}$ (Arjmand et al., 2006; Verla et al., 2012; Romero-Anaya et al., 2014), $\mathrm{K}_{2} \mathrm{CO}_{3}$ (Foo et Hameed, 2012), $\mathrm{Na}_{2} \mathrm{CO}_{3}$ (Verla et al., 2012) sont également utilisées comme agents d'activation chimique. Certains chercheurs ont rapportés que la propension de la méthode d'activation par voie chimique au $\mathrm{H}_{3} \mathrm{PO}_{4}$ et $\mathrm{ZnCl}_{2}$ est liée à sa faible température d'activation (400-500 $\left.{ }^{\circ} \mathrm{C}\right)$ par rapport à l'activation physique (supérieur à $850^{\circ} \mathrm{C}$ ), à des temps de traitement plus courts et la meilleure qualité des charbons actifs obtenus; (Guo et Rockstraw, 2007; Prauchner et Rodriguez-Reinoso, 2008; Arami-Niya et al., 2010; Demiral et al., 2011). Par ailleurs, le rendement des charbons actifs préparés par activation chimique est d'environ 3,7 fois plus grand que ceux obtenus par la méthode d'activation physique (Altenor et al., 2009). L'activation chimique génère des charbons actifs avec un meilleur développement des mésopores (Prauchner et Rodriguez-Reinoso, 2008). Particulièrement, l'activation chimique avec $\mathrm{H}_{3} \mathrm{PO}_{4}$ donne des charbons actifs avec une meilleure efficacité pour l'élimination des micropolluants organiques et inorganiques en raison d'un meilleur développement de leur porosité, leurs surfaces spécifiques et le volume des pores (Ioannidou et Zabaniotou, 2007; Prauchner et Rodriguez-Reinoso, 2008). En outre, l'activation chimique avec $\mathrm{H}_{3} \mathrm{PO}_{4}$ présente d'autres avantages tels que: la possibilité de modification des groupes fonctionnels de surface et la structure des pores; le changement de la structure de texture (Nowicki et al., 2010); une remarquable capacité d'échange d'ions et une stabilité chimique aussi bien à des températures élevées que dans les solutions aqueuses acides et basiques (Puziy et al., 2002; Wang et Zhu, 2007; Attia et al., 2008).

Le mécanisme d'action de l'acide phosphorique sur le précurseur tel que rapporté par Ahmed et al. (2007); Guo et 
Rockstraw (2006) comporte deux étapes : (i) la décomposition pyrolytique du précurseur ; (ii) la formation d'une structure réticulée. La décomposition pyrolytique est favorisée par l'effet catalytique du $\mathrm{H}_{3} \mathrm{PO}_{4}$ sur les réactions de clivage de liaison. Par contre, le phénomène réticulé est dû à des interactions entre la biomasse et $\mathrm{H}_{3} \mathrm{PO}_{4}$, conduisant à la formation des liaisons phosphates entre les fragments dans la biomasse.

L'objectif de ce travail est de valoriser les coques de noix de coco béninoise en charbon actif capable d'adsorber des micropolluants dans les eaux. Le travail consiste à préparer des charbons actifs par voie chimique à l'acide phosphorique $\mathrm{H}_{3} \mathrm{PO}_{4}$, en suivant l'effet de certains paramètres de préparation (ratio d'imprégnation, taux de dilution de l'acide phosphorique et température de carbonisation) sur l'indice d'iode et sur les propriétés texturales (surface spécifique et distribution des pores) des différents charbons actifs obtenus.

\section{MATERIEL ET METHODES Matériels}

Les coques de noix de coco collectées dans un marché local au Bénin ont été grattées pour enlever les fibres. Elles sont broyées et tamisées (Tamis AFNOR) pour retenir les particules de tailles comprises entre 2 à 2,5 $\mathrm{mm}$. Elles sont ensuite lavées abondamment à l'eau distillée puis séchées encore à l'étuve (Instruments Heraeus) à $110^{\circ} \mathrm{C}$ pendant $24 \mathrm{~h}$. Les particules séchées ont été soumises à une activation chimique à l'acide phosphorique.

\section{Méthode de préparation des charbons actifs}

Le procédé comprend deux étapes. La première consiste à imprégner $6 \mathrm{~g}$ de coques de noix de coco par une solution d'acide orthophosphorique avec des concentrations $\left(\mathrm{V}_{\text {acide }} / \mathrm{V}_{\text {solution }}\right)$ allant de 12,5 à $50 \%$ et un ratio d'imprégnation $\mathrm{Xp}$ variant entre 0,7 et 2,5 gramme d'acide par gramme de coque de noix de coco. Le mélange d'imprégnation est chauffé à reflux, puis filtré et séché à $110^{\circ} \mathrm{C}$ dans une l'étuve. Les particules ainsi obtenues sont carbonisées dans un four (Nabertherm LE14 C290/11) pendant $2 \mathrm{~h}$ à des températures de 500, 600 et $700{ }^{\circ} \mathrm{C}$, c'est la deuxième étape. Après refroidissement à température ambiante dans un dessiccateur, le charbon actif obtenu est lavé d'abord avec une solution $0,1 \mathrm{~N}$ d'acide chlorhydrique puis rincé plusieurs fois avec de l'eau distillée chaude jusqu'à pH constant.

\section{Analyse \\ Rendement}

Le rendement du charbon actif préparé a été estimé à partir de l'équation suivante:

$$
\operatorname{Rca}(\mathbf{w t} \%)=\frac{\text { masse du charbon actif }(\mathrm{g})}{\text { masse de coque de noix de coco }(\mathrm{g})} \mathrm{X} 100
$$

Rca $=$ Rendement de charbon actif

Indice d'iode des charbons actifs

L'indice d'iode des charbons actifs préparés a été déterminé en utilisant la norme ASTM D4607-94 (2006). Selon cette norme, la quantité d'iode adsorbé (en mg) par gramme de carbone activé avec une concentration résiduelle d'iode de $0,02 \mathrm{~N}$, représente l'indice d'iode. La méthode se présente comme suit :

a) $50 \mathrm{ml}$ d'une solution d'iode $(0,1 \mathrm{~N})$ est ajoutée à trois différentes masses d'échantillons de charbon actif contenu dans trois Erlenmeyer de $250 \mathrm{ml}$, initialement mouillé avec $5 \mathrm{ml}$ de $\mathrm{HCl}$ à $5 \%$;

b) le contenu de la fiole est agité vigoureusement pendant $30( \pm 1)$ secondes, puis filtré rapidement à travers un papier filtre plissé Whatman, $\mathrm{N}^{\circ} 2 \mathrm{~V}$.

c) $25 \mathrm{ml} \mathrm{du}$ filtrat est titrée par une solution de thiosulfate de sodium $0,1 \mathrm{~N}$ jusqu'à ce que la solution devienne jaune pâle. $2 \mathrm{ml}$ d'une solution d'indicateur à l'amidon (1 $\mathrm{g} / \mathrm{l})$ ont été ajoutés, et le dosage a été poursuivi avec du thiosulfate de sodium jusqu'à ce que la solution devienne incolore. On note $\mathrm{V}_{2}$, le volume de thiosulfate consommé.

d) on détermine la quantité d'iode adsorbée, exprimée en mg par gramme de charbon actif pour chacune des trois masses, soit $\mathrm{X} / \mathrm{m}_{\mathrm{CA}}$ 
$\frac{\mathrm{X}}{\mathrm{m}_{\mathrm{CA}}}=\frac{126.9044}{\mathrm{~m}_{\mathrm{CA}}}\left(50 . \mathrm{N}_{1}-55 . \mathrm{C}_{\mathrm{r}}\right)$

avec

$C_{r}=\frac{N_{2} V_{2}}{V}$

où:

- $\mathrm{C}_{\mathrm{r}}$, la concentration en d'iode résiduel du filtrat;

- $\mathrm{N}_{1}$, la normalité de la solution de la solution d'iode;

- $\mathrm{N}_{2}$, la normalité de la solution de thiosulfate;

- V, le volume du filtrat titré $(\mathrm{V}=25 \mathrm{ml})$

Pour déterminer l'indice d'iode, on trace la courbe $\mathrm{X} / \mathrm{m}_{\mathrm{CA}}=\mathrm{f}\left(\mathrm{C}_{\mathrm{r}}\right)$ en utilisant les trois masses. Cette courbe est une droite. L'indice d'iode correspond à la valeur $\mathrm{X} / \mathrm{m}_{\mathrm{CA}}$ pour laquelle la concentration en iode résiduel est $0,02 \mathrm{~N}$.

Caractérisation de la texture: surface spécifique et structure poreuse

La texture des charbons actifs a été déterminée par adsorption d'azote $\mathrm{N}_{2}$ à $77 \mathrm{~K}$. Cette mesure a été effectuée avec le porosimètre multi gaz ASAP $2010 \mathrm{M}$. La surface spécifique $\left(S_{\text {B.E.T }}\right)$ a été déterminée en utilisant l'équation de Brunauer, Emmett et Teller (B.E.T). Le volume poreux total $\mathrm{V}_{\mathrm{T}}$, a été défini comme le volume d'azote liquide correspondant à la quantité adsorbée à la pression relative la plus élevée de $\mathrm{P} / \mathrm{P}_{0}=0,99$ (Basta et al., 2009). Le volume microporeux, $\mathrm{V} \mu$, a été déterminé par la méthode d'analyse micro pores (MP). Le volume des mésopores $\left(\mathrm{V}_{\text {meso }}\right)$ a été déterminé par la méthode Barrett, Joyner, et HalendaBJH (Guo et Rockstraw, 2006). Le diamètre moyen des pores, Dp, a été calculé en utilisant la relation $4 \mathrm{~V}_{\mathrm{T}} / \mathrm{S}_{\mathrm{BET}}$, et la distribution de taille des pores, par la méthode MP-Brunaeur (Basta et al., 2009).

\section{Caractéristiques chimiques}

Les caractéristiques chimiques (fonctions chimiques de surfaces, $\mathrm{pH}$ au point de charges nulle) des charbons actifs préparés ont été déterminées respectivement à partir des méthodes de Boehm et de Lopez-Ramon et al. (Boehm, 1966; Lopez-Ramon et al., 1999). Pour la détermination qualitative des fonctions de surface, nous avons utilisé un spectromètre Infrarouge Tensor 27 (Bruker) avec une résolution de $4 \mathrm{~cm}^{-1}$. La ligne de base est faite avec une pastille de $\mathrm{KBr}$ et les mesures sont faites sur les poudres.

\section{RESULTATS}

Effet du ratio d'imprégnation et du taux de dilution sur l'indice d'iode

Il a été procédé à des mesures de l'indice d'iode des charbons actifs obtenus suivant différents ratios d'imprégnation Xp et diverses concentrations d'acide phosphorique pour une carbonisation à $500{ }^{\circ} \mathrm{C}$ pendant $2 \mathrm{~h}$. Les valeurs de l'indice d'iode varient entre 432 à $787 \mathrm{mg}^{-g^{-1}}$ tel que le montre la Figure 1. L'allure des courbes de cette figure montre que pour un même taux de dilution, l'indice d'iode augmente lorsque le ratio d'imprégnation Xp passe de 0,7 à 2 avec des valeurs maximales pour le taux de dilution de $50 \%$. Mais, en passant du ratio d'imprégnation $\mathrm{Xp}, 2$ à 2,5, nous avons observé une diminution de l'indice d'iode pour cette même dilution de $50 \%$ et la température de carbonisation de $500{ }^{\circ} \mathrm{C}$.

S'agissant de l'effet de dilution, on observe globalement que pour un ratio d'imprégnation donné et à la température de carbonisation de $500{ }^{\circ} \mathrm{C}$, l'indice d'iode croît avec le taux de dilution. Toutefois, on note une légère diminution lorsque le taux de dilution augmente de $16,7 \%$ à $25 \%$. Ceci est plus remarquable avec le ratio d'imprégnation $\mathrm{Xp}=2,5$. La concentration en $\mathrm{H}_{3} \mathrm{PO}_{4}$ optimum pour l'activation est de $50 \%$. En outre, il est observé, pour le ratio d'imprégnation $\mathrm{Xp}=1,5$, une augmentation sensible de l'indice d'iode qui passe de 663 à $786 \mathrm{mg} / \mathrm{g}$ lorsque taux de dilution passe de $33,33 \%$ à $50 \%$.

Effet du ratio d'imprégnation et de la concentration d'acide sur le rendement massique

La Figure 2 présente les variations du rendement et d'indice d'iode en fonction du 
ratio d'imprégnation des charbons actifs pour une concentration d'acide phosphorique de $50 \%$ et la température de carbonisation de 500 ${ }^{\circ} \mathrm{C}$. Le rendement varie de $29,74 \%$ à $38,96 \%$ pour des ratios d'imprégnation $\mathrm{Xp}$ compris entre 0,7 et 2 . Outre, le ratio d'imprégnation $\mathrm{Xp}=0,7$ qui présente un faible rendement, on n'observe pas un effet remarquable de la variation du ratio d'imprégnation sur le rendement avec les paramètres fixes de température $\left(500{ }^{\circ} \mathrm{C}\right)$ et le taux de dilution de $50 \%$ de l'acide.

Dans les conditions: ratio d'imprégnation à l'acide $\mathrm{Xp}=1,5$ et à la température de carbonisation de $500{ }^{\circ} \mathrm{C}$, l'effet de la concentration sur le rendement a été déterminé. La Figure 3 montre des rendements massique allant de 32,5 à $41,4 \%$ pour des taux de dilution d'acide compris entre 12,5 et $50 \%$.

\section{Propriétés texturales}

Effet du ratio d'imprégnation à l'acide sur l'adsorption de $\mathrm{N}_{2}$ et la porosité

La Figure 4 montre les isothermes d'adsorption de $\mathrm{N}_{2}$ des charbons actifs obtenus pour des ratios d'imprégnation $\mathrm{Xp}$ allant de 0,7 à 2,0 avec le taux de dilution d'acide phosphorique de $50 \%$ et la même température de carbonisation de $500{ }^{\circ} \mathrm{C}$. On observe sur cette figure, des capacités d'adsorption les plus élevées et un début de boucle d'hystéris aux pressions relatives $\mathrm{P} / \mathrm{P}_{0}$ proches de 1 pour des charbons actifs préparés avec des ratios d'imprégnation Xp 1,5 et 2,0. L'isotherme du charbon actif de ratio d'imprégnation $\mathrm{xp}=$ 0,7 , présente un plateau horizontal pour des pressions relatives $\mathrm{P} / \mathrm{P}_{0}$ supérieures à 0,2 . Ce charbon présente la plus faible capacité d'adsorption d'azote $\mathrm{N}_{2}$. La surface spécifique de ce charbon actif $(\mathrm{Xp}=0,7)$ est la plus petite (Figure 10), $\mathrm{S}_{\mathrm{BET}}=1264,0 \mathrm{~m}^{2} \cdot \mathrm{g}^{-1}$. Il est donc de type I.

Toutes les caractéristiques texturales et l'indice d'iode des charbons actifs préparés avec un taux de dilution d'acide fixé à $50 \%$ aussi bien pour divers ratio d'imprégnation
(Xpallant de 0,7 à 2) que pour différentes températures $\left(500\right.$ à $700{ }^{\circ} \mathrm{C}$ ) avec un ratio d'imprégnation fixe de 1,5 , sont regroupées dans le Tableau 1.

Effet de la température sur la capacité d'adsorption de $\mathrm{N}_{2}$ et la porosité

La Figure 5 donne les isothermes d'adsorption de $\mathrm{N}_{2}$ à $77 \mathrm{~K}$, des charbons actifs obtenus pour un même ratio d'imprégnation $\mathrm{Xp}=1,5$ avec le taux de dilution d'acide phosphorique de $50 \%$ et des températures de carbonisation de 500, 600 et $700{ }^{\circ} \mathrm{C}$. Les isothermes d'adsorption de l'azote $\mathrm{N}_{2}$ pour ces trois charbons de différentes températures de carbonisation, ont même forme à des pressions $\mathrm{P} / \mathrm{P}_{0}$ relativement faibles $(<0,3)$. A des pressions relatives $\mathrm{P} / \mathrm{P}_{0}$ supérieures à 0,3 , le charbon actif ayant été carbonisé à $500{ }^{\circ} \mathrm{C}$ présente la plus grande capacité d'adsorption de $\mathrm{N}_{2}$ avec une forte augmentation de la pente de son isotherme. Par contre, la pente des deux charbons (avec température de carbonisation de 600 et $700{ }^{\circ} \mathrm{C}$ ) croît faiblement. Donc une augmentation de la température diminue la capacité d'adsorption de l'azote des charbons actifs. Le Tableau 1 et la Figure 10 montrent l'augmentation de la microporosité avec la température. Le rapport $\mathrm{V}_{\mu} / \mathrm{V}_{\mathrm{T}}$ croît avec la température. Quant à la surface spécifique, elle reste presque constante en passant de $500{ }^{\circ} \mathrm{C}$ à la température supérieure mais marquée toutefois par une très faible diminution.

Les caractéristiques chimiques des charbons actifs

Détermination de $\mathrm{pH}$ au point de charge nulle $\left(\mathrm{pH}_{\mathrm{ZPC}}\right)$

La Figure 11 présente la courbe de détermination $\mathrm{du} \mathrm{pH}_{\mathrm{ZPC}}$ pour le charbon actif au ratio d'imprégnation $\mathrm{Xp}=1,5$ au taux de dilution d'acide de $50 \%$ et à la température de carbonisation de $500{ }^{\circ} \mathrm{C}$. La valeur du $\mathrm{pH}_{\mathrm{ZPC}}$ pour ce charbon actif est de 3,16 . 
Détermination des groupes fonctionnels de surface par la méthode de Boehm

Les résultats de la détermination des groupes de surface par la méthode de Boehm pour tester l'échantillon du charbon actif au ratio d'imprégnation $\mathrm{Xp}=1,5$ avec un taux de dilution d'acide de $50 \%$ indiquent la présence de groupes carboxyle, lactones et quinones (carbonyle) avec les valeurs $(\mathrm{mEq} / \mathrm{g})$, respectivement de 2,0; 1,25 et 1,5.
Analyse par spectroscopie IR-FT des groupes fonctionnels de surface

La Figure 12 montre le spectre FT-IR des charbons actifs préparés avec des ratios d'imprégnation allant de 0,7 à 2 , au taux de dilution de $50 \%$. On observe pour ces quatre charbons actifs, trois principales bandes d'absorption (1220, 1590 et $3378 \mathrm{~cm}^{-1}$ ).

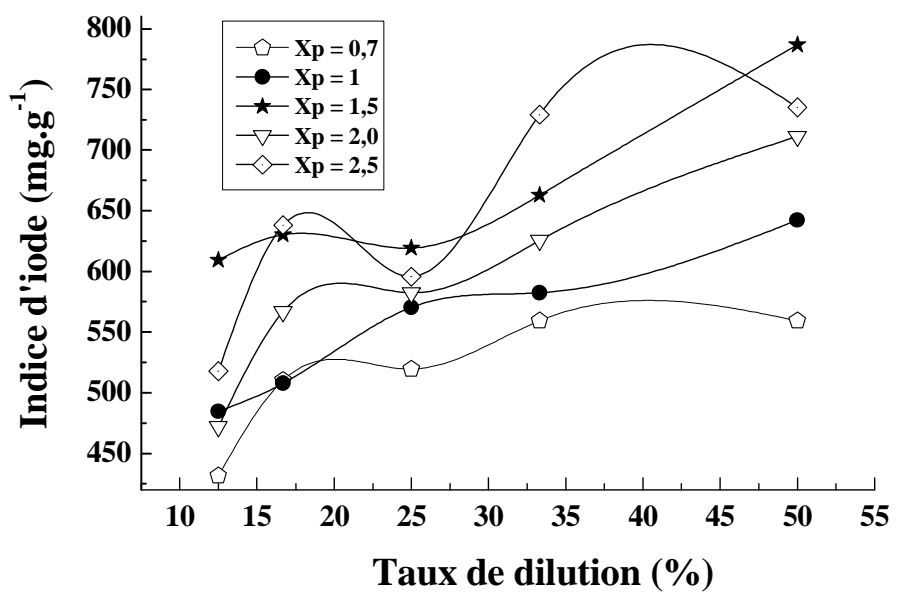

Figure 1: Effet de la dilution sur l'indice d'iode des charbons actifs pour différents ratios d'imprégnation Xp à la température de carbonisation de $500{ }^{\circ} \mathrm{C}$.

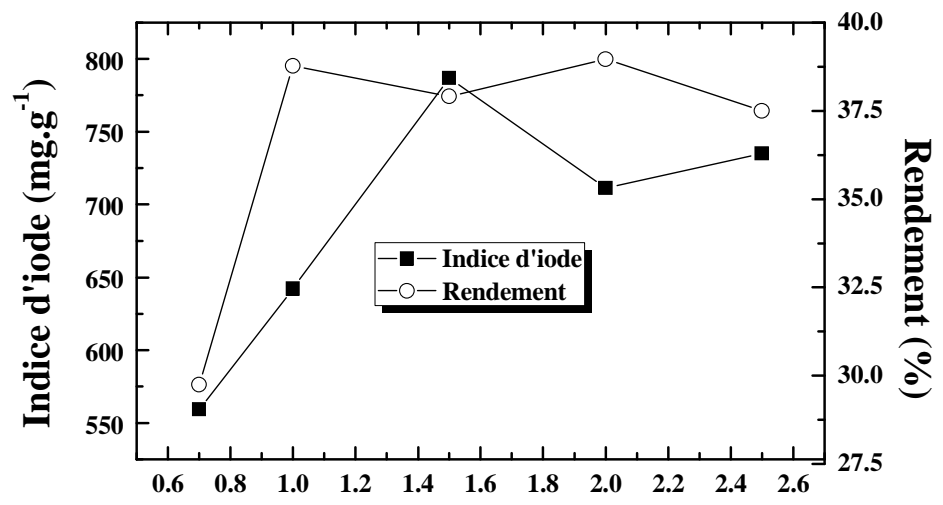

Ratio d'imprégnation Xp

Figure 2: Effet du ratio d'imprégnation sur l'indice d'iode et le rendement du charbon actif pour un taux de dilution de $50 \%$ à la température de carbonisation de $500{ }^{\circ} \mathrm{C}$. 


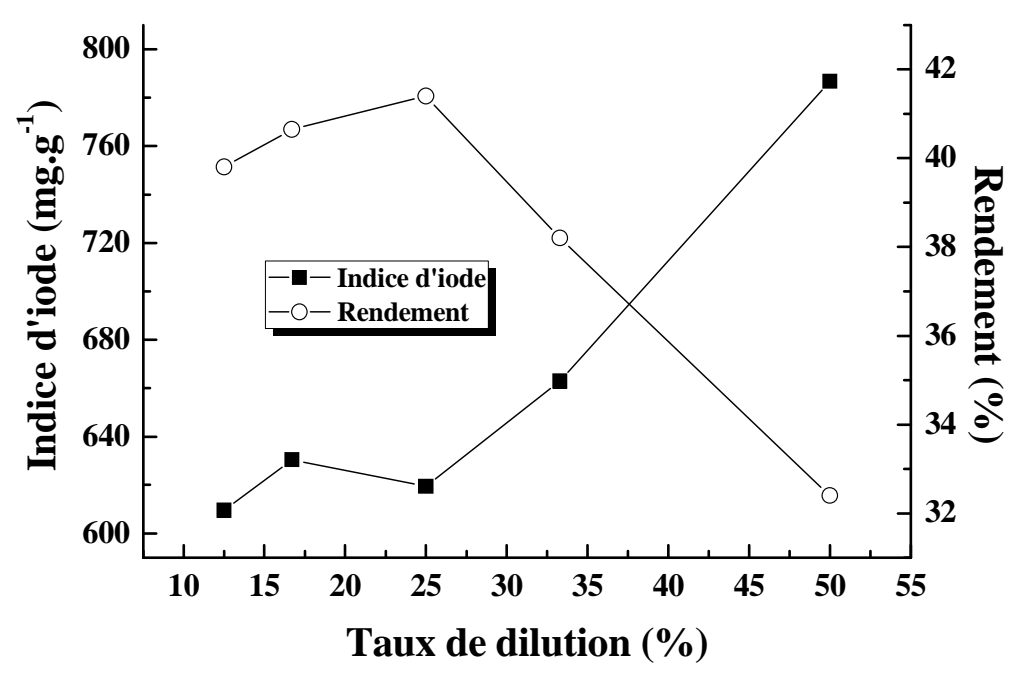

Figure 3: Effet du taux de dilution sur le rendement et l'indice d'iode du charbon actif pour $\mathrm{Xp}=1,5$ à la température de carbonisation de $500^{\circ} \mathrm{C}$.

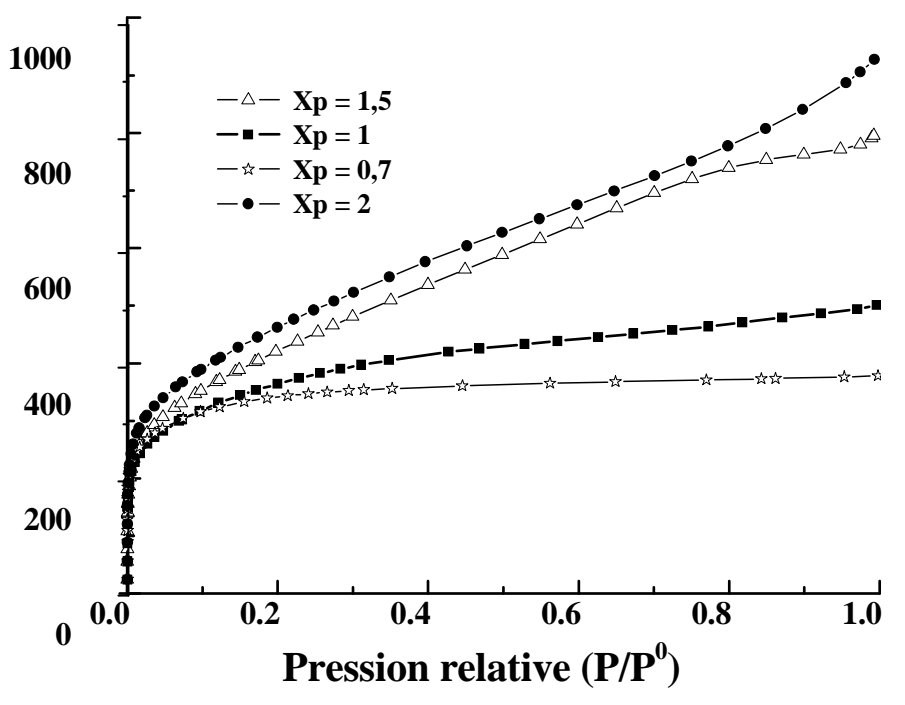

Figure 4: Isothermes d'adsorption de $\mathrm{N}_{2}$ et les CA pour différentes températures de carbonisation et pour différents ratios d'imprégnation au $\mathrm{H}_{3} \mathrm{PO}_{4}, \mathrm{Xp}$ à $\mathrm{T}=500{ }^{\circ} \mathrm{C}$. 


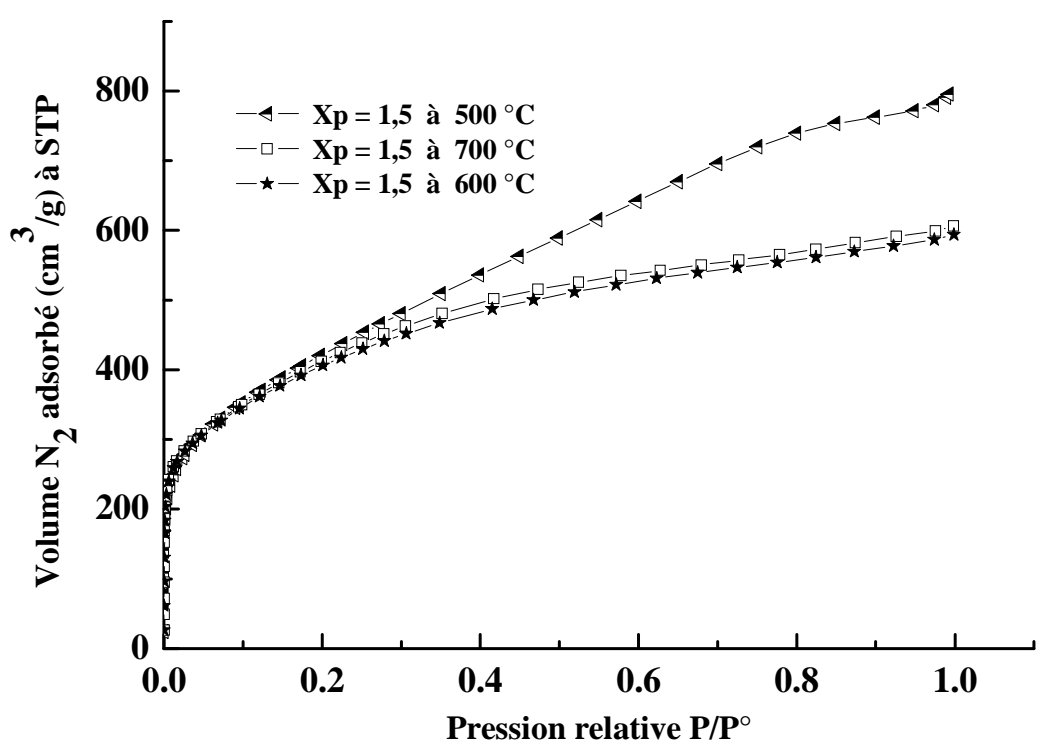

Figure 5: Isothermes d'adsorption de l'azote $\mathrm{N}_{2}$ avec un ratio d'imprégnation $\mathrm{Xp}=1,5$, taux de dilution de $50 \%$ pour des températures de carbonisation de $500{ }^{\circ} \mathrm{C}, 600{ }^{\circ} \mathrm{C}$ et $700{ }^{\circ} \mathrm{C}$.

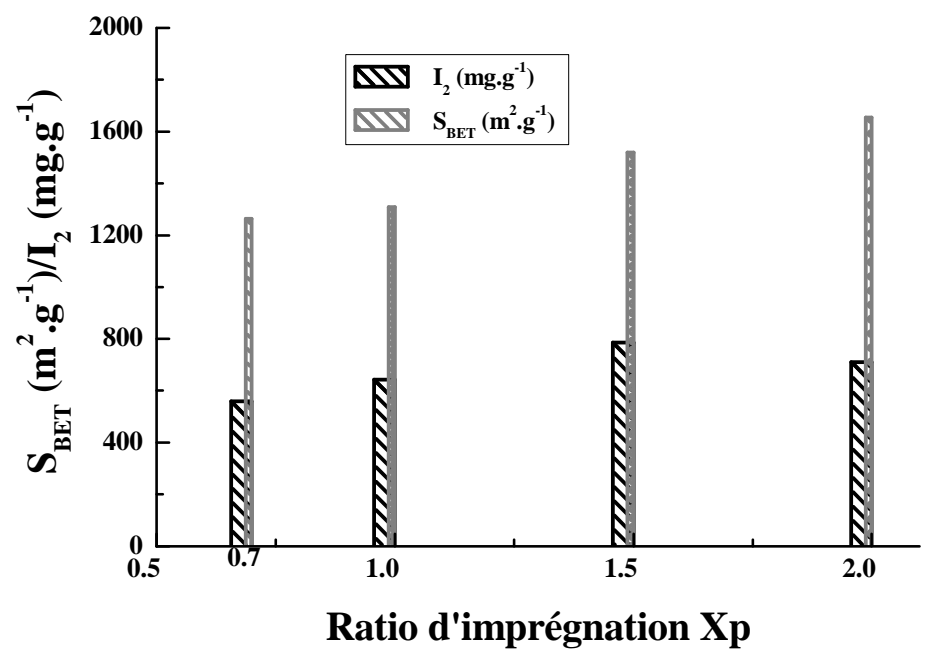

Figure 6: Effet du ratio d'imprégnation sur le développement des micros et mésopores pour un ratio d'imprégnation $\mathrm{Xp}=1,5$, taux de dilution de $50 \%$ et température de carbonisation de $500{ }^{\circ} \mathrm{C}$. 


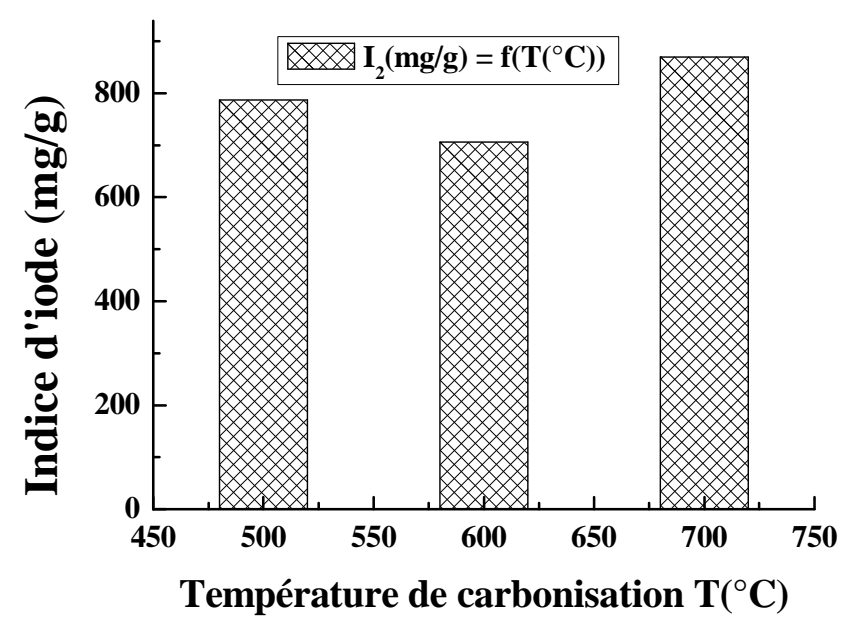

Figure 7: Effet de la température de carbonisation sur l'indice d'iode pour $\mathrm{Xp}=1,5$ et taux de dilution de $50 \%$.

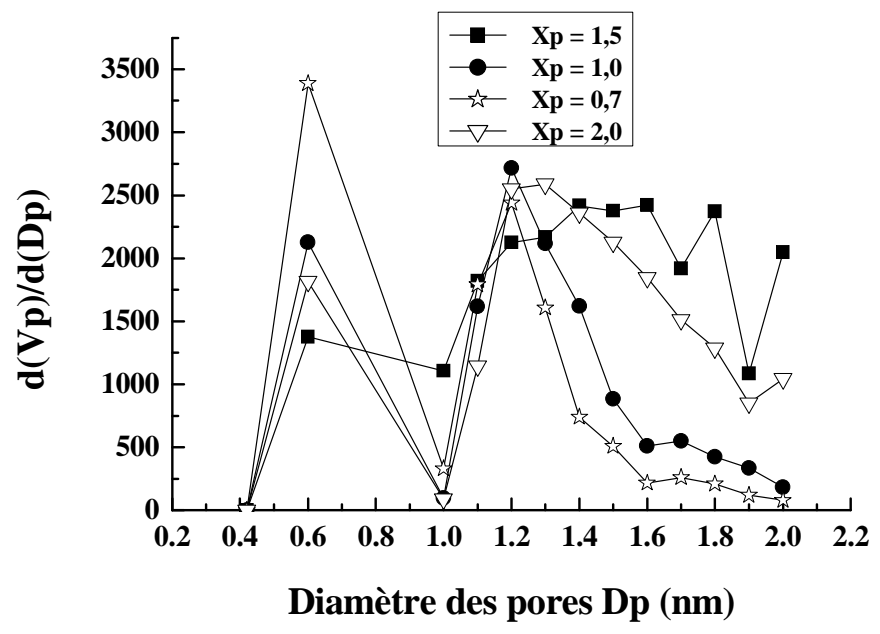

Figure 8: Distribution des tailles des micropores des charbons actifs à différents ratios d'imprégnation Xp pour un taux de dilution d'acide de $50 \%$ et à la température de $500{ }^{\circ} \mathrm{C}$. 


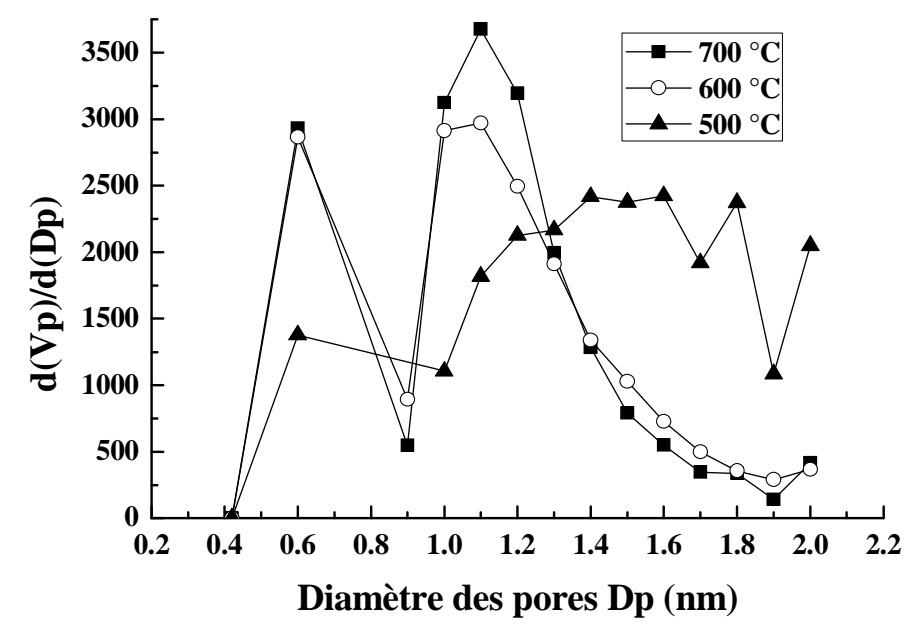

Figure 9: Distribution de la taille du micropore des charbons actifs pour $\mathrm{Xp}=1,5$ à différentes températures de carbonisation.

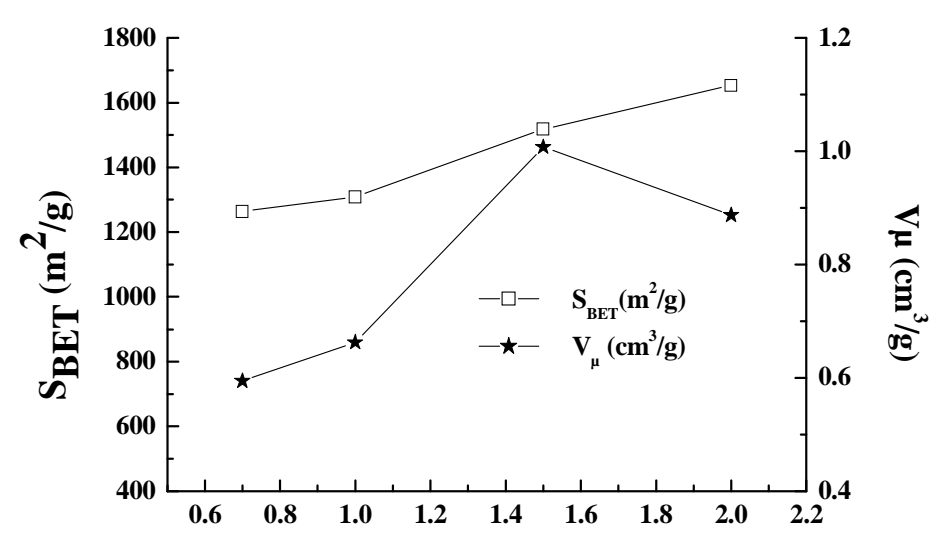

Ratio d'imprégnation Xp

Figure 10: Surface spécifique et volume du micropore pour différents ratios d'imprégnation Xp au taux de dilution de $50 \%$ et la température de $500{ }^{\circ} \mathrm{C}$. 


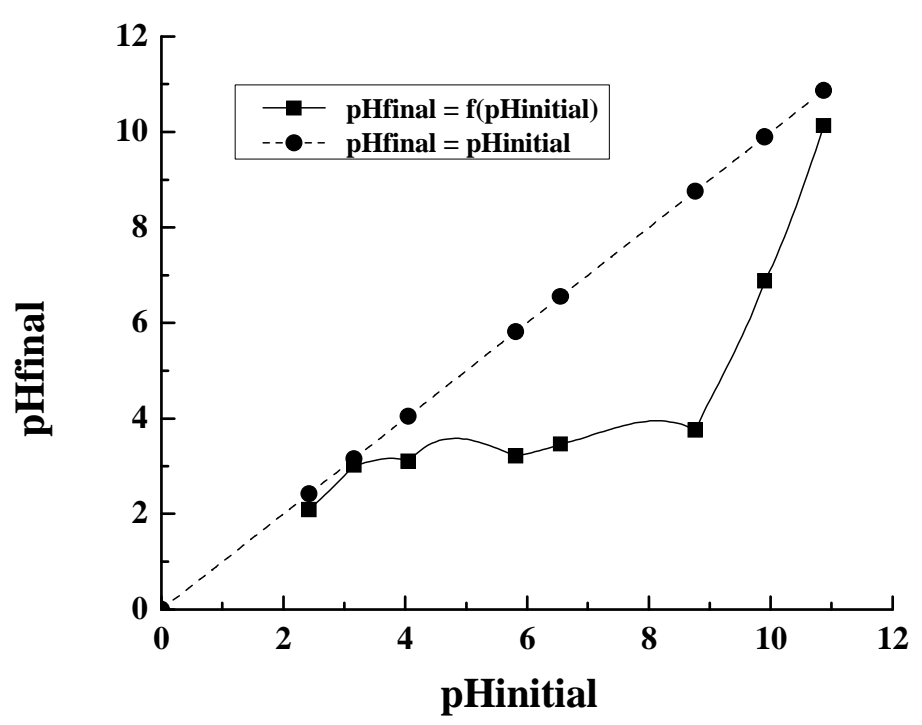

Figure 11: Courbe de détermination du $\mathrm{pH}_{\mathrm{ZPC}}$.

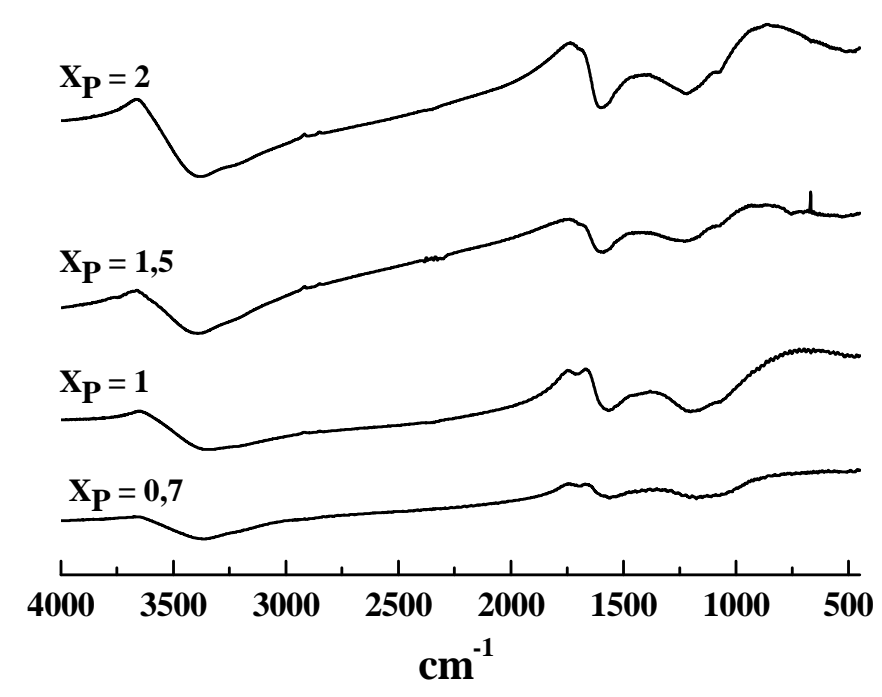

Figure 12: Spectre IR-FT pour les charbons actifs à différents ratios d'imprégnation Xp pour un taux de dilution d'acide de $50 \%$ et à la température de $500^{\circ} \mathrm{C}$. 
Tableau 1: Propriétés texturales et indice d'iode de quelques charbons actifs.

\begin{tabular}{|c|c|c|c|c|c|c|c|c|c|}
\hline \multirow[t]{2}{*}{$\mathbf{C A}$} & \multirow[t]{2}{*}{$\mathbf{X p}$} & \multirow{2}{*}{$\begin{array}{c}\mathbf{t} \\
\left({ }^{\circ} \mathbf{C}\right)\end{array}$} & \multirow{2}{*}{$\begin{array}{c}\mathbf{I}_{2} \\
(\mathrm{mg} / \mathrm{g})\end{array}$} & \multicolumn{4}{|l|}{ BETBJH } & $\frac{\text { MP- runauer }}{V}$ & \multirow{2}{*}{$\begin{array}{l}\mathbf{V}_{\mu} / \mathbf{V} \\
T(\%)\end{array}$} \\
\hline & & & & $\begin{array}{c}\mathrm{S}_{\mathrm{BET}} \\
\left(\mathrm{m}^{2 \cdot} \mathbf{g}^{-1}\right) \\
\end{array}$ & $\begin{array}{c}\mathbf{V}_{\mathbf{T}} \\
\left(\mathrm{cm}^{3} \cdot \mathrm{g}^{-1}\right)\end{array}$ & $\begin{array}{c}\text { Dp } \\
\text { (nm) }\end{array}$ & $\begin{array}{c}\mathbf{V}_{\text {méso }} \\
\left(\mathbf{c m}^{3} \cdot \mathbf{g}^{-1}\right)\end{array}$ & $\begin{array}{c}\mathbf{V}_{\mu} \\
\left(\mathrm{cm}^{3} \cdot \mathrm{g}^{-1}\right)\end{array}$ & \\
\hline $\mathrm{CNC}_{-1}$ & 0,7 & 500 & 559 & 1264,0 & 0,5843 & 1,850 & 0,1236 & 0,5943 & 102 \\
\hline $\mathrm{CNC}_{-2}$ & 1,0 & 500 & 642 & 1309,2 & 0,7714 & 2,357 & 0,3788 & 0,6624 & 85,9 \\
\hline $\mathrm{CNC}_{-3}$ & 1,5 & 500 & 787 & 1519,0 & 1,2234 & 3,221 & 0,9514 & 1,0079 & 82,4 \\
\hline $\mathrm{CNC}_{-4}$ & 2,0 & 500 & 736 & 1653,7 & 1,4280 & 3,454 & 1,0978 & 0,8870 & 62,1 \\
\hline $\mathrm{CNC}_{\mathrm{T} 1}$ & 1,5 & 600 & 706 & 1448,6 & 0,9139 & 2,524 & 0,5320 & 0,8102 & 88,65 \\
\hline $\mathrm{CNC}_{\mathrm{T} 2}$ & 1,5 & 700 & 870 & 1464,3 & 0,9332 & 2,549 & 0,5550 & 0,8156 & 87,4 \\
\hline
\end{tabular}

\section{DISCUSSION}

La qualité des charbons actifs a été appréciée par les mesures d'indice d'iode et la détermination de leurs propriétés texturales (surface spécifique, porosité et distribution de la taille des pores). L'effet des paramètres : concentration de l'acide phosphorique, ratio d'imprégnation et température, a été déterminé par les mesures des indicateurs (indice d'iode et adsorption de l'azote $\mathrm{N}_{2}$, porosité et surface spécifique). Ce qui a permis de déterminer conditions d'optimisation du procédé avec un bon rendement.

\section{Effet de la concentration d'acide et du ratio d'imprégnation sur l'indice d'iode}

Il a été établi que l'indice d'iode donne une estimation de la surface spécifique, et il mesure la porosité des pores de dimension $\geq$ $1,0 \mathrm{~nm}$ (Haimour et Emeish, 2006; Girgis et El-Hendawy, 2002). L'indice d'iode est une indication de la capacité d'adsorption dans les micropores (Verla et al., 2012; Sun et Jiang, 2010). Il existe donc une corrélation avérée entre l'indice d'iode et la surface spécifique.

Il ressort de la Figure 2 que l'augmentation du ratio d'imprégnation engendre sensiblement le développement des micropores, entrânant ainsi une augmentation de la capacité d'adsorption des charbons actifs et donc l'indice d'iode. Mais lorsque le ratio dépasse 2 grammes d'acide par gramme de précurseur, une partie des micropores formés sont élargis ou détruits du fait de la capacité oxydante très élevée de l'acide phosphorique
$\mathrm{H}_{3} \mathrm{PO}_{4}$. Cette diminution du nombre de micropores s'accompagne d'une réduction sensible de la capacité d'adsorption du charbon actif. Il apparaît donc qu'au-delà du ratio d'imprégnation $\mathrm{Xp}=2$, l'acide phosphorique ne semble plus être bénéfique pour l'augmentation de l'indice d'iode. L'indice d'iode maximum de $787 \mathrm{mg} \cdot \mathrm{g}^{-1}$, correspond au ratio d'imprégnation $\mathrm{Xp}=1,5$ et un taux de dilution d'acide de $50 \%$ pour la température de carbonisation de $500{ }^{\circ} \mathrm{C}$. Ce résultat est semblable à ceux des travaux menés sur les noyaux de datte (Girgis et ElHendawy, 2002) et sur les biomasses ligneuses de bouleau (Budinova et al., 2006). Par contre, pour les pousses de mûrier élagué, bien que l'optimum de capacité d'adsorption d'iode corresponde à la concentration d'acide phosphorique de $50 \%$, le ratio d'imprégnation pour cet optimum d'indice d'iode est égal à 2,5 (Wang et al., 2010).

Les résultats montrent que la concentration de $\mathrm{H}_{3} \mathrm{PO}_{4}$ influence fortement la capacité d'adsorption d'iode $\mathrm{I}_{2}$ des échantillons. Ce paramètre apparaît donc comme un facteur clé qui affecte l'indice d'iode du charbon actif préparé, tel que rapporté par Prahas et al. (2008). Les valeurs relativement élevées des indices d'iode des charbons actifs produits au taux de dilution à l'acide de $50 \%$ et à la température de carbonisation de $500{ }^{\circ} \mathrm{C}$, constituent donc un bon indicateur de la microporosité desdits matériaux et de leurs grandes surfaces spécifiques. 
Effet du ratio d'imprégnation à l'acide sur le rendement massique

Le rendement de charbon actif est une mesure importante de la faisabilité de l'élaboration de charbon actif à partir d'un précurseur donné et dans des conditions déterminées. C'est un indicateur de la performance d'une méthode de préparation de ce matériau surtout à l'échelle industrielle. Les travaux effectués par Guo et Rockstraw (2006) en utilisant la xylose, la cellulose, et la lignine pour la préparation du charbon actif par voie chimique à l'acide phosphorique, ont montré que d'importantes différences de rendement du produit peuvent être observées en fonction du précurseur et les conditions de préparation (proportion de l'agent d'imprégnation, la température de carbonisation et d'activation).

Le rendement massique maximum des charbons préparés est de $38,96 \%$. Hormis le charbon actif au ratio d'imprégnation $\mathrm{Xp}=$ 0,7 , le rendement massique est peu sensible à la variation du ratio d'imprégnation Xp allant de 1 à 2,5 (Figure 2). En effet, les rendements massiques obtenus pour ces ratios sont presque constant avec une moyenne d'environ $38 \%$. Les rendements des charbons actifs, pour cette étude sont comparables aux résultats des travaux antérieurs effectués par deux groupes de chercheurs sur les coques de noix de coco qui sont de $32,46 \%$ (FFTCAPR, 2004). Une valeur semblable $(38,12 \%)$ de rendement a été rapportée par Wang et al. (2010) pour la préparation de charbon actif à partir des pousses de mûrier élagué.

\section{Caractéristiques texturales des charbons actifs \\ - Effet du ratio d'imprégnation sur les isothermes d'adsorption de $N_{2}$, la surface spécifique et la distribution de taille des pores}

La distribution de taille des pores (DTP) détermine la fraction du volume total des pores accessible à des molécules de taille et de forme données. La capacité d'adsorption des charbons actifs est la plus importante des propriétés directement liées à la surface spécifique et au volume des pores (Nowicki et al., 2010).

Les isothermes d'adsorption de $\mathrm{N}_{2}$ (Figure 4) des charbons actifs préparés en faisant varié le ratio d'imprégnation à l'acide avec température de carbonisation et taux de dilution d'acide fixe, sont de type I et de type II selon la classification de Brunauer, Deming, Deming et Teller, classification BDDT (Tien, 1994). L'isotherme de type I représente l'adsorption monomoléculaire et ne s'applique qu'à des adsorbants microporeux avec de petites tailles de pores. L'isotherme de type II caractérise les adsorbants présentant une large gamme de tailles de pores de telle sorte que l'adsorption peut s'étendre de la monocouche au multicouche et en fin de compte à la condensation capillaire (Yavuz et al., 2010). Il ressort donc de nos résultats que les charbons obtenus dans les conditions sus indiquées sont constituées majoritairement des micropores pour les ratios Xp 1,5 et 2,0 à la température de carbonisation de $500{ }^{\circ} \mathrm{C}$. Mais le début de boucle d'hystéris observée à des pressions relatives proches de 1 au niveau de leurs isothermes indique l'apparition de petits mésopores. La diminution de la capacité d'adsorption de $\mathrm{N}_{2}$ lorsque la température augmente pour les charbons $\mathrm{Xp}=1,5$ et taux de dilution de $50 \%$ pourrait être justifiée par l'élargissement des pores défavorable à l'adsorption de $\mathrm{N}_{2}$ surtout pour des pores de grand diamètre (macropores). Ces résultats sont en accord avec ceux des études menées par certains chercheurs ( Baçaoui et al., 2001; Budinova et al., 2006; Guo et Rockstraw, 2007) sur différents types de biomasses activées à l'acide phosphorique qui indiquent que les propriétés texturales des charbons actifs sont influencées par les ratios d'imprégnation et la température de carbonisation.

Pour ce groupe de charbon actif (Xp variable et les autres paramètres fixes), l'augmentation de la surface spécifique $\mathrm{S}_{\mathrm{BET}}$ de 1264,0 à $1653,7 \mathrm{~m}^{2} \cdot \mathrm{g}^{-1}$ lorsque le ratio d'imprégnation passe de 0,7 à 2 se justifierait par un développement de la porosité. Par contre, pour l'indice d'iode, on observe 
qu'après croissance pour les ratios allant de 0,7 à 1,5 ; celui-ci diminue en passant de 787 mg.g à 736 mg. $\mathrm{g}^{-1}$ pour le ratio d'imprégnation de 2. Ceci est lié à un agrandissement des pores car le rapport $\mathrm{V}_{\mu} / \mathrm{V}_{\mathrm{T}}$ égal à $62,1 \%$ pour $\mathrm{Xp}=2$ est inférieur à celui du ratio d'imprégnation $\mathrm{Xp}=1,5(82,4 \%)$. Ce qui confirme que le volume micropores a chuté au profit des pores de plus grands diamètres (mésopores et macropores). Des groupes de chercheurs ont pu remarquer que l'augmentation de ratio d'imprégnation à l'acide phosphorique engendre la diminution de la microporosité (Girgis et El-Hendawy, 2002; Wang et al., 2010). Aussi, en observant la Figure 7, on constate une augmentation du volume des mésopores alors que le volume des micropores diminue lorsque le ratio d'imprégnation passe de 1,5 à 2 . Pour cette variation du ratio, la proportion du volume des micropores par rapport au volume total des pores passe de 82,4 à $62,1 \%$ avec aussi une augmentation du diamètre moyen des pores Dp qui passe de 3,22 à 3,45 nm. Des résultats semblables qui indiquent que l'augmentation du ratio d'imprégnation pour l'activation par voie chimique permet d'améliorer le développement de pores, ont été rapportés par certains chercheurs (Prahas et al., 2008; Lua et al., 2004; Baçaoui et al., 2001). En effet, l'augmentation du ratio engendre sensiblement le développement des micropores.

La distribution dans le domaine de la microporosité des charbons actifs préparés au ratio d'imprégnation allant de $\mathrm{Xp}=0,7$ à 2 au taux de dilution de $50 \%$ et à la température de carbonisation $500{ }^{\circ} \mathrm{C}$ (figure 8), signale pour ces quatre charbons actifs, la présence remarquée des pores de diamètre $0,6 \mathrm{~nm}$ donc de l'ultramicropore (diamètre des pores $<0,7$ nm) (Arami-Niya et al., 2010). Cette ultramicroporosité est plus élevée pour le charbon actif correspondant au ratio d'imprégnation $\mathrm{Xp}=0,7$ et plus faible pour celui au ratio $\mathrm{Xp}=1,5$. Par contre, le charbon actif (avec $\mathrm{Xp}=1,5$ ), présente le volume le plus élevé des pores de diamètre allant de 0,9 à $2 \mathrm{~nm}$. Le pic dans le domaine des micropores pour le charbon actif obtenu au ratio $\mathrm{Xp}=1,5$, correspond à un diamètre $\mathrm{Dp}$ de 1,60 $\mathrm{nm}$ contre le plus faible diamètre des micropores $\mathrm{Dp}=0,60 \mathrm{~nm}$ pour le charbon actif au ratio d'imprégnation $\mathrm{Xp}=0,7$. Ces résultats justifient et confirment la plus forte valeur d'indice d'iode obtenue pour ce charbon actif à ratio d'imprégnation $\mathrm{Xp}=1,5$, taux de dilution d'acide de $50 \%$ avec une température de carbonisation de $500{ }^{\circ} \mathrm{C}$, car l'iode est adsorbé dans les pores de diamètre $\geq$ $1 \mathrm{~nm}$ (Haimour et Emeish, 2006; Girgis et ElHendawy, 2002).

\section{Effet de la température sur la texture poreuse des charbons actifs}

La Figure 7 permet d'apprécier l'effet de la température sur l'indice d'iode des charbons obtenus par imprégnation à la solution d'acide phosphorique diluée à 50\% et un ratio $\mathrm{Xp}=1,5$. En faisant passer la température de carbonisation de 500 à $700{ }^{\circ} \mathrm{C}$, l'indice d'iode augmente de 787 à $870 \mathrm{mg} . \mathrm{g}^{-1}$ avec une légère diminution à $600{ }^{\circ} \mathrm{C}$. Les surfaces spécifiques $\mathrm{S}_{\mathrm{BET}}$ mesurées diminuent de 1519,0 à $1448,6 \mathrm{~m}^{2} / \mathrm{g}$. La valeur la plus faible $\left(1448,6 \mathrm{~m}^{2} / \mathrm{g}\right)$ dans ce cas correspond à la température de carbonisation de $600{ }^{\circ} \mathrm{C}$.

L'augmentation de la température a favorisé l'élargissement des pores, mais aussi l'apparition de nouveaux ultramicropores en témoigne les valeurs des pics de $d(V p) / d\left(d_{P}\right)$ observés aux environs de $d_{P} 0,6 \mathrm{~nm}$ qui passent de 1376 à 2866. Ceci justifie la diminution du diamètre moyen des micropores qui passe de 1,60 à $1,10 \mathrm{~nm}$ lorsque la température de carbonisation passe de 500 à $700{ }^{\circ} \mathrm{C}$. Par ailleurs, l'élargissement des pores en mésopores et macropores justifierait la diminution du volume microporeux. La valeur maximum ( $\left.870 \mathrm{mg} \cdot \mathrm{g}^{-1}\right)$ de l'indice d'iode pour cette catégorie de charbons actifs correspond à la température de carbonisation de $700{ }^{\circ} \mathrm{C}$. Ceci est compatible avec la distribution des pores présentée sur la Figure 10 ou le pic le plus élevé de $d(V p) / d(D p)$ dans le domaine des micropores, se situe au diamètre de pore de $1,1 \mathrm{~nm}$. Aussi le rapport $\mathrm{V}_{\mu} / \mathrm{V}_{\mathrm{T}}$ égal à 87,4 pour le charbon actif carbonisé à $700{ }^{\circ} \mathrm{C}$, qui 
est supérieur à $(82,4 \%)$ pour celui carbonisé à $500{ }^{\circ} \mathrm{C}$. Les ultramicropores auraient probablement évolué beaucoup plus vers les micropores sous l'effet de l'augmentation de la température. Ceci engendre une augmentation de la capacité d'adsorption de ce charbon vis-à-vis de l'iode. Cette observation est en accord avec les résultats des travaux de certains chercheurs qui ont indiqué que l'indice d'iode augmente sensiblement au fur et à mesure que la température augmente (Aygün et al., 2003; Girgis et El-Hendawy, 2002).

Les caractéristiques chimiques des charbons actifs au taux de dilution d'acide de $50 \%$ et à différents ratios d'imprégnation

- Analyse de la chimie de surface par spectroscopie FT-IR et méthode de Boehm

La bande à environ $3378 \mathrm{~cm}^{-1} \mathrm{de}$ la Figure 12 peut être attribuée à des vibrations des groupes hydroxyles O-H. Le spectre des fréquences comprises entre $1703 \mathrm{~cm}^{-1}$ et 1590 $\mathrm{cm}^{-1}$ peut être attribué à l'étirement symétrique de $\mathrm{C}=\mathrm{C}$ et de $\mathrm{C}=\mathrm{O}$ des groupements pyrone, aldéhyde ou carboxylique (Shen et al., 2008; Chianget al., 2002). Les Signaux 1292 et $1278 \mathrm{~cm}^{-1}$ correspondant à La liaison $\mathrm{C}-\mathrm{O}$ des phénols, acide carboxylique, ester et/ou à la liaison $\mathrm{P}=$ $\mathrm{O}$ des phosphates d'ester, ou à la liaison $\mathrm{O}-\mathrm{C}$ de $\mathrm{P}-\mathrm{O}-\mathrm{C}$, ou à la liaison $\mathrm{P}=\mathrm{OOH}$ (Puziy et al., 2005). Les Signaux 1130 et $1160 \mathrm{~cm}^{-1}$ correspondant aux composés phosphoreux (Puziy et al., 2005). Enfin, la bande autour de $1220 \mathrm{~cm}^{-1}$ est liée aux vibrations de $\mathrm{C}=\mathrm{O}$ et C-O (Shen et al., 2008; Chianget al., 2002). Pour les charbons activés à l'acide phosphorique, l'augmentation du ratio d'imprégnation, jusqu'à une valeur de 2 , a conduit à une augmentation importante de toutes les bandes d'absorption, ce qui indique une plus forte contribution des groupes de surface à l'apparition des groupements oxygénés. La présence des groupes hydroxyle, carbonyle et des composés aromatiques est une preuve de la structure ligno-cellulosique de la coque de noix de coco telle que rapportée par les résultats des travaux de préparation et de caractérisation des charbons actifs par activation chimique à l'acide phosphorique par certains chercheurs (Al Bahri, 2012) qui ont travaillé sur la noix de coco et les graines de raisin respectivement. L'interprétation des résultats de FT-IR indique la présence de groupes acide carboxylique, phénolique et de pyrone. La dominance $\mathrm{du}$ groupe fonctionnel carboxylique à la surface à partir des résultats obtenus en utilisant la méthode de Boehm justifierait la valeur de $\mathrm{pH}_{\mathrm{ZPC}}$. Ce résultat est conforme à celui obtenu par Khelifi et al. (2009). $\mathrm{H}_{3} \mathrm{PO}_{4}$, en tant qu'oxydant fort, il peut oxyder des atomes de surface et leur fait perdre des électrons pour être chargés positivement. L'oxygène au même moment captant ces électrons serait chargé négativement et pourrait se fixer sur les oxydes de surface (Lopez et al., 1999).

- $\quad$ H au point de charge nulle $\left(\mathrm{pH}_{Z P C}\right)$

Le $\mathrm{pH}$ au point de charge nulle $\left(\mathrm{pH}_{\mathrm{ZPC}}\right)$ est un paramètre très important qui indique le comportement acide-base des solides. Dans les études de sorption, c'est un paramètre utile qui permet de faire l'hypothèse sur l'ionisation des groupes fonctionnels à la surface de charbons actifs et de leurs interactions avec les adsorbats. Au $\mathrm{pH}_{\mathrm{ZPC}}$, la charge des sites de surface positive est égale à celle des sites de surface négative (Martínez de Yuso et al., 2014). La valeur de 3,16 du $\mathrm{pH}_{\mathrm{ZPC}}$ indique le caractère acide de ce charbon actif (Vargas et al., 2011; Puziy et al., 2002). Des valeurs semblables $\left(\mathrm{pH}_{\mathrm{ZPC}}=3,2\right.$ et 3,4$)$ ont été rapportées par Song et al. (2010); Altenor et al. (2009) pour des charbons actifs à base de coque de noix de coco oxydés à l'acide nitrique et des racines de vétiver préparés par activation au $\mathrm{H}_{3} \mathrm{PO}_{4}$, respectivement.

\section{Conclusion}

Les résultats de cette étude indiquent qu'il est possible de préparer de bons charbons actifs avec des caractéristiques structurales intéressantes en agissant sur les paramètres d'activation. L'étude expérimentale qui a consisté à suivre 
l'influence du ratio d'imprégnation, de la température de carbonisation et de la concentration de l'acide phosphorique a permis d'obtenir du charbon actif ayant le maximum d'indice d'iode et de surface BET, essentiellement microporeux avec des volumes de micropores représentant plus de $82 \%$ du volume total des pores dans les conditions où le ratio d'imprégnation $\mathrm{Xp}=1,5$ et un taux de dilution de $50 \%$ avec une température d'activation de $500{ }^{\circ} \mathrm{C}$ pendant $2 \mathrm{~h}$. Les analyses effectuées ont montré que les propriétés texturales ainsi que la chimie de surface déterminée respectivement par l'analyse de la physisorption $\mathrm{N}_{2}$ et FTIR et les techniques de caractérisation primaires (détermination de l'indice d'iode et de la méthode de Boehm) sont concordants. Les valeurs élevées d'indices d'iode des charbons actifs préparés traduisent un pouvoir adsorbant intéressant. Il apparaît clairement que certains charbons actifs produits dans cette étude sont susceptibles d'être utilisés dans le traitement des eaux.

\section{REFRENCES}

Ahmed Hared I, Dirion JL, Salvador S, Lacroix M, Rio S. 2007. Pyrolysis of wood impregnated with phosphoric acid for the production of activated carbon: kinetics and porosity development studies. J Anal Appl Pyrolysis., 79(1): 101-105.

Al Bahri M, Calvo L, Gilarranz MA, Rodriguez JJ. 2012. Activated carbon from grape seeds upon chemical activation with phosphoric acid: Application to the adsorption of diuron from water. Chemical Engineering Journal., 203: 348-356.

Altenor S, carene B, Emmanuel E,Lambert J, Ehrhardt JJ, Gaspard S. 2009. Adsorption studies of methylene blue and phenol onto vetiver roots activated carbon prepared by chemical activation. Journal of Hazardous Materials., 165(1-3): 10291039.

Arami-Niya A, Wan Daud WMA, Mjalli FS.2010. Production of palm shell-based activated carbon with more homogeniouse pore size distribution. $J$. Applied Sci., 10(24): 3361-3366.
Arjmand C, Kaghazchi T, Seyed ML, Soleimani M. 2006. Chemical Production of Activated Carbon from Nutshells and Date Stones. Chem. Eng. Technol., 29(8): 986-991.

ASTM D4607-94. 2006. Standard Test Method for Determination of Iodine Number of Activated Carbon. ASTM International 100 Barr Harbor Drive: United States.

Attia AA, Girgis BS, Fathy NA. 2008. Removal of methylene blue by carbon derived from peach stones by $\mathrm{H}_{3} \mathrm{PO}_{4}$ activation: batch and column studies. Dyes Pigments., 76(1): 282-289.

Aygün A, Yenisoy-Karakaş S, Duman I. 2003. Production of granular activated carbon from fruit stones and nutshells and evaluation of their physical, chemical and adsorption properties. Microporous and Mesoporous Materials.,66(2-3): 189-195.

Baçaoui A, Yaacoubi A, Dahbi A, Bennouna C, Phan Tan Luu R, Maldonado-Hodar F J, Rivera-Utrilla J, Moreno-Castilla C. 2001. Optimisation of conditions for the preparation of activated carbons from olive-waste cakes. Carbon., 39(3): 425432.

Basta AH, Fierro V, El-SaiedH, CelzardA.2009. Steps KOH activation of rice straw: an efficient method for preparing high-performance activated carbons. Bioresour. Technol., 100(17): 3941-3947.

Boehm H. 1966. Chemical Identification of Surface Groups. Academic Press: London; 179-274.

Budinova T, Ekinci E, Yardim F, Grimm A, Björnbom E, Minkova V, Goranova M.2006.Characterization and application of activated carbon produced by $\mathrm{H}_{3} \mathrm{PO}_{4}$ and water vapor activation. Fuel Processing Technology., 87(10): 899-905.

Cazetta LA, Junior PO, Vargas MMA, da Silva PA, Zou X, Asefa T, Almeida CV. 2013. Thermal regeneration study of high surface area activated carbon obtained from coconut shell: Characterization and application of 
response surface methodology. Journal of Analytical and Applied Pyrolysis., 101: 53-60.

Chiang HL, Huang CP, Chiang PC. 2002. The surface characteristics of activated carbon as affected by ozone and alkaline treatment. Chemosphere, 47(3): 257-265.

Chitou L. 2003. Effets des différents types de compost à base d'ordures ménagères de Cotonou sur la production du chou pommé sur sol minéral brut du littoral. Mémoire d'Ingénieur des Travaux. Ecole Polytechnique d'Ab-Calavi, Université d'Ab-Calavi UAC, Abomey-calavi, Bénin, p. 16.

COMDEV et l'Association Internationale pour le Commerce et le Développement Durable AICDD. 2010. Symposium international sur la gestion durable et la valorisation des déchets dans les pays en développement, Cotonou.

Demiral H, Demiral I, Karabacakoglu B, Tümsek F.2011. Production of activated carbon from olive bagasse by physical activation. Chemical Engineering Research and Design., 89(2): p. 206-213.

FFTCAPR (Food and Fertilizer Technology Center for the Asian and Pacific Region). 2004. Processing of coconut shell into activated carbon/charcoal. Document.

Foo KY, Hameed BH. 2012. Preparation, characterization and evaluation of adsorptive properties of orange peel based activated carbon via microwave induced $\mathrm{K}_{2} \mathrm{CO}_{3}$ activation. Bioresource Technology.,104: 679-686.

Girgis BS, El-Hendawy A A. 2002. Porosity development in activated carbons obtained from date pits under chemical activation with phosphoric acid. Micropor. Mesopor. Mater., 52(2): 105117.

Guo Y, Rockstraw DA. 2006. Physical and chemical properties of carbons synthesized from xylan, cellulose, and Kraft lignin by $\mathrm{H}_{3} \mathrm{PO}_{4}$ activation. Carbon., 44(8): 1464-1475.

Guo Y, Rockstraw DA. 2007. Physicochemical properties of carbons prepared from pecan shell by phosphoric acid activation. Bioresource Technology., 98(8): 1513-1521.

Haimour NM, Emeish S. 2006. Utilization of date stones for production of activated carbon using phosphoric acid. Waste Management., 26(6): 651-660.

Ioannidou O, Zabaniotou A. 2007. Agricultural residues as precursors for activated carbon production-A review. Renewable and Sustainable Energy Reviews., 11(9): 1966-2005.

Ji YB, Li TH, Zhu L, Wang XX, Lin QL. 2007. Preparation of activated carbons by microwave heating $\mathrm{KOH}$ activation. Appl. Surf. Sci., 254(2): 506-512.

Khelifi A, Temdrara L, Addoun A. 2009. Effet de la texture poreuse et de la structure chimique sur l'adsorption du bleu de méthylène par des charbons actifs oxydés. J. Soc. Alger. Chim., 19(1): 1325.

Lopez-Ramon MV, StoeckliF, MorenoCastilla C, Carrasco-MarinF.1999. On the characterization of acidic and basic surface sites on carbons by various techniques. Carbon, 37(8): 1215-1221.

Lua AC, Yang T, Guo J. 2004. Effects of pyrolysis conditions on the properties of activated carbons prepared from pistachio-nut shells. J. Anal. Appl. Pyrol., 72: 279-287.

Martínez de Yuso A, Rubio B, Teresa Izquierdo M. 2014. Influence of activation atmosphere used in the chemical activation of almond shell on the characteristics and adsorption performance of activated carbons. Fuel Processing Technology., 119: 74-80.

Nowicki P, Pietrzak R, Wachowska H.2010.Sorption properties of active carbons obtained from walnut shells by chemical and physical activation. Catalysis Today.,150(1-2): 107-114.

Prauchner MJ, Rodriguez-Reinoso F. 2008. Preparation of granular activated carbons for adsorption of natural gas. Microporous Mesoporous Mater., 109(13): 581-584. 
Prahas D, Kartika Y, Indraswati N, Ismadaji S. 2008. Activated carbon from jackfruit peel waste by $\mathrm{H}_{3} \mathrm{PO}_{4}$ chemical activation: pore structure and surface chemistry characterization. Chem. Eng. J., 140(1-3): 32-42.

Puziy AM, Poddubnaya OI, Martinez-Alonso A, Suarez-Garcia F, Tascon JMD. 2002. Synthetic carbons activated with phosphoric acid I. Surface chemistry and ion binding properties. Carbon, 40(9): 1493-1505.

Puziy AM, Poddubnaya OI, Martinez-Alonso A, Suarez-Garcia F, Tascon JMD. 2005. Surface chemistry of phosphoruscontaining carbons of lignocellulosic origin. Carbon, 43(14): 2857-2868.

Romero-Anaya AJ, Ouzzine M, LilloRódenas MA, Linares-Solano A. 2014. Spherical carbons: Synthesis, characterization and activation processes. Carbon, 68: 296-307.

Sahu JN, Acharya J, Meikap BC. 2010. Optimization of production conditions for activated carbons from tamarind wood by zinc chloride using response surface methodology. Bioresour. Technol., 101(6): 1974-1982.

Sartape A, Mandhare A, Salvi P, Pawar D, Raut P, Anuse M, Kolekar S. 2012.Removal of Bi (III) with Adsorption Technique Using Coconut Shell Activated Carbon. Chinese Journal of Chemical Engineering, 20(4): 768-775.

Shen WZ, Li ZJ, Liu YH.2008. Surface chemical functional groups modification of porous carbon. Recent Patents Chem. Eng., 1(1): 27-40.

Song X, Liu H, Cheng L, Qu Y. 2010. Surface Modification of Coconut-Based Activated Carbon by Liquid-Phase Oxidation and Its Effects on Lead Ion Adsorption. Desalination, 255(1): 78-83.

Sun K, Jiang JC. 2010. Preparation and characterization of activated carbon from rubber seed shell by physical activation with steam. Biomass Bioenerg., 34(4): 539-544.

Tsai WT, Chang CY, Wang SY, Chang CF, Chien SF, Sun HF. 2001. Preparation of activated carbon from corn cob catalyzed by potassium salts and subsequent gasification with $\mathrm{CO}_{2}$. Bioresource Technology., 78(2): 203-208.

Tien C. 1994. Adsorption Calculations and Modeling. Butterworth-Heinemannseries in Chemical Engineering: Boston.

Vargas AMM, Cazetta AL, Garcia CA, Moraes JCG, Nogami EM, Lenzi E, Costa WF, Almeida VC. 2011. Preparation and characterization of activated carbon from a new raw lignocellulosic material: Flamboyant (Delonix regia) pods. Journal of Environmental Management., 92(1): 178184.

Verla AW, Horsfall (Jnr) M, Verla EN, Spiff AI, Ekpete OA.2012. Preparation and Characterization of Activated Carbon from fluted PUMPKIN (TELFAIRIA OCCIDENTALIS HOOK.F) seed shell. Asian Journal of Natural \& Applied Sciences, 1(3): 39-50.

Wang J, Wu FA, Wang M, Qiu N, LiangY, Shui-Qin Fang SQ, Jiang X.2010. Preparation of activated carbon from a renewable agricultural residue of pruning mulberry shoot. African Journal of Biotechnology, 19(9): 2762-2767

Wang S, Zhu ZH. 2007. Effects of acidic treatment of activated carbons on dye adsorption. Dyes Pigments., 75(2): 306314

Yavuz R, Akyıldız H, Karatepe N, Çetinkaya E. 2010. Influence of preparation conditions on porous structures of olive stone activated by $\mathrm{H}_{3} \mathrm{PO}_{4}$. Fuel Processing Technology, 91(1): 80-87. 